Keywords: urinary bladder neoplasms; neutrophils; lymphocytes; biological markers; prognosis; treatment outcome; cystectomy; inflammation

\title{
Pre-treatment neutrophil-to-lymphocyte ratio as predictor of adverse outcomes in patients undergoing radical cystectomy for urothelial carcinoma of the bladder
}

\author{
T Hermanns ${ }^{\star}, 1,2,6$, B Bhindi ${ }^{2,3,6}, Y \mathrm{Wei}^{1}$, J Yu ${ }^{1}$, A P Noon ${ }^{1,2}, \mathrm{P} O$ Richard $^{1,2}$, J R Bhatt ${ }^{1,2}$, A Almatar ${ }^{1,2}$,
} M A S Jewett ${ }^{1,2}$, N E Fleshner ${ }^{1,2}$, A R Zlotta ${ }^{2,4}$, A J Templeton ${ }^{5}$ and G S Kulkarni ${ }^{1,2,3}$

${ }^{1}$ Division of Urology, Department of Surgical Oncology, Princess Margaret Cancer Centre, 610 University Avenue, Toronto, Ontario, Canada M5G 2M9; ${ }^{2}$ Division of Urology, Department of Surgery, University Health Network, University of Toronto, 610 University Avenue, Toronto, Ontario, Canada M5G 2M9; ${ }^{3}$ Institute for Clinical Evaluative Sciences, 2075 Bayview Avenue, Toronto, Ontario, Canada M4N 3M5; ${ }^{4}$ Department of Urology, Mount Sinai Hospital, 60 Murray Street, Toronto, Ontario, Canada M5T 3L9 and ${ }^{5}$ Division of Medical Oncology, Department of Medicine, Princess Margaret Cancer Centre, 610 University Avenue, Toronto, Ontario, Canada M5G 2M9

Background: An elevated neutrophil-to-lymphocyte ratio (NLR) is associated with poor outcome in various tumours. Its prognostic utility in patients with urothelial carcinoma of the bladder (UCB) undergoing radical cystectomy (RC) is yet to be fully elucidated.

Methods: A cohort of patients undergoing RC for UCB in a tertiary referral centre between 1992 and 2012 was analysed. Neutrophil-to-lymphocyte ratio was computed using complete blood counts performed pre-RC, or before neo-adjuvant chemotherapy where applicable. Time-dependent receiver operating characteristic curves were used to determine the optimal cutoff point for predicting recurrence-free survival (RFS), cancer-specific survival (CSS) and overall survival (OS). The predictive ability of NLR was assessed using Kaplan-Meier analyses and multivariable Cox proportional hazards models. The likelihood-ratio test was used to determine whether multivariable models were improved by including NLR.

Results: The cohort included 424 patients followed for a median of 58.4 months. An NLR of 3 was determined as the optimal cutoff value. Patients with an NLR $\geqslant 3.0$ had significantly worse survival outcomes (5y-RFS: $53 \%$ vs $64 \%$, log-rank $P=0.013 ; 5 y$-CSS: $57 \%$ vs $75 \%$, log-rank $P<0.001 ; 5 y-O S: 43 \%$ vs $64 \%$, log-rank $P<0.001)$. After adjusting for disease-specific predictors, an NLR $\geqslant 3.0$ was significantly associated with worse RFS ( $H R=1.49 ; 95 \% \mathrm{Cl}=1.12-2.0, P=0.007), \mathrm{CSS}(\mathrm{HR}=1.88 ; 95 \% \mathrm{Cl}=1.39-2.54, P<0.001)$ and OS (average $\mathrm{HR}=1.67 ; 95 \% \mathrm{Cl}=1.17-2.39, P=0.005$ ). The likelihood-ratio test confirmed that prognostic models were improved by including NLR.

Conclusions: Neutrophil-to-lymphocyte ratio is an inexpensive prognostic biomarker for patients undergoing RC for UCB. It offers pre-treatment prognostic value in addition to established prognosticators and may be helpful in guiding treatment decisions.

Radical cystectomy (RC) with pelvic lymph node dissection is the standard treatment for muscle-invasive (MI) urothelial carcinoma of the bladder (UCB) and is recommended for patients with non-muscle-invasive (NMI) UCB with high risk of progression (Clark et al, 2013). Despite curative intent, disease recurs in a significant proportion of patients and 5-year survival

\footnotetext{
*Correspondence: Dr T Hermanns; E-mail: thomas.hermanns@uhn.ca

${ }^{6}$ These authors contributed equally to this work.
}

Received 20 February 2014; revised 18 April 2014; accepted 12 May 2014; published online 10 June 2014 
rates of only $40-60 \%$ have consistently been reported (Gakis et al, 2013).

More aggressive treatment options, such as early RC in patients with high-risk NMI UCB or RC in combination with neo-adjuvant chemotherapy (NAC) or adjuvant chemotherapy (AC), have been shown to improve outcomes (Raj et al, 2011; Meeks et al, 2012; Leow et al, 2013; Sternberg et al, 2013). However, employing aggressive strategies unselectively to all patients carries the risk of overtreatment in patients with favourable prognoses. Improved risk stratification will individualise the use of such approaches. At this time, however, risk stratification based on clinicopathological data alone is unlikely to be sufficient for optimal treatment decision-making (Ficarra et al, 2005; Shariat et al, 2007; Canter et al, 2011). Thus, novel prognostic markers are needed to improve stratification, and, eventually outcomes, of patients with UCB.

Inflammation has an important role in the development and progression of many malignancies (Grivennikov et al, 2010; Hanahan and Weinberg, 2011). Putative mechanisms include the increased supply of factors that promote carcinogenesis and tumour progression by cells of the innate immune systems (that is, neutrophils) and decreased antitumoural response by immune cells of the adaptive system (that is, lymphocytes; Hanahan and Weinberg, 2011). The neutrophilto-lymphocyte ratio (NLR), which can easily be calculated from routine complete blood counts (CBCs) with differentials, is an emerging marker of host inflammation and has been shown to be an independent prognosticator for a variety of solid malignancies (Proctor et al, 2012; Guthrie et al, 2013; Templeton et al, 2014). However, there is sparse data on the prognostic role of NLR in patients with UCB (Gondo et al, 2012; Demirtas et al, 2013; Krane et al, 2013).

The objective of our investigation was to evaluate the association between pre-treatment NLR and survival in patients undergoing RC for UCB in a cohort of patients from a tertiary care centre.

\section{MATERIALS AND METHODS}

Patients and data sources. Using our institutional database, patients who underwent RC between 1 January 1992 and 31 December 2012 were retrospectively identified. Patients were excluded if CBCs with differentials were unavailable for analysis $(n=14)$, or if they had a history of conditions that may have influenced blood cell lines (connective tissue disease: $n=4$, malignant lymphoma: $n=3$, leukaemia: $n=2$, and human immunodeficiency virus infection: $n=1$ ). Patients undergoing $\mathrm{RC}$ for salvage therapy following failed chemoradiation $(n=20)$ were excluded because of the potential influence of prior chemotherapy on blood cell lines. Patients with non-urothelial cancers $(n=9)$, or for primary prostatic urothelial carcinoma $(n=5)$, were also excluded in order to maintain a homogenous cohort. Electronic hospital chart review was performed to collect

Table 1. Cohort characteristics

\begin{tabular}{|c|c|c|c|c|c|}
\hline & & $\begin{array}{c}\text { Total } \\
n=424\end{array}$ & $\begin{array}{c}\text { NLR } \geqslant 3 \\
n=216\end{array}$ & $\begin{array}{c}\text { NLR }<3 \\
n=208\end{array}$ & $P$-value \\
\hline \multicolumn{6}{|l|}{ Patient characteristics } \\
\hline $\begin{array}{l}\text { Age in years } \\
\text { Female sex, } n \\
\text { Haemoglobin }\left(\mathrm{gl}^{-1}\right) \\
\text { WBC }\left(\times 10^{9} \mathrm{I}^{-1}\right) \\
\text { Platelets }\left(\times 10^{9} \mathrm{I}^{-1}\right) \\
\text { Heavy smoking }(30 \geqslant \text { pack-years }), n \\
\text { Charlson comorbidity index }\end{array}$ & & $\begin{aligned} 70.1 & (60.6-76.3) \\
99 & (23.4) \\
132.5 & (116-145) \\
7.6 & (6.0-9.1) \\
252 & (202-303) \\
134 & (31.6) \\
6 & (5-7)\end{aligned}$ & $\begin{aligned} 71.5 & (61.9-77.3) \\
43 & (20.7) \\
125 & (109-140) \\
8.7 & (7.05-10.4) \\
274 & (218-329) \\
64 & (30.8) \\
6 & (5-8)\end{aligned}$ & $\begin{aligned} 68.9 & (59.5-75.5) \\
56 & (25.9) \\
137.5 & (125-148.5) \\
6.6 & (5.5-8.2) \\
236 & (193-282.5) \\
70 & (32.4) \\
6 & (4.5-7)\end{aligned}$ & $\begin{aligned} & 0.086 \\
& 0.20 \\
< & 0.001^{\mathrm{a}} \\
< & 0.001^{\mathrm{a}} \\
< & 0.001^{\mathrm{a}} \\
& 0.72 \\
& 0.010^{\mathrm{a}}\end{aligned}$ \\
\hline \multicolumn{6}{|l|}{ Disease characteristics } \\
\hline $\begin{array}{l}\text { Hydronephrosis, n (\%) } \\
\text { Concurrent CIS, n (\%) } \\
\text { T-stage, } n(\%) \\
\text { N-stage, } n(\%) \\
\text { Positive surgical margin, } n(\%) \\
\text { Lymphovascular invasion }\end{array}$ & $\begin{array}{c}\text { pT0 } \\
\text { pTa/T1/T2 CIS } \\
\text { pT3/T4 } \\
\text { pNO } \\
\text { pN-pos } \\
\text { pNx }\end{array}$ & $\begin{array}{c}119(28.1) \\
190(44.8) \\
25(5.9) \\
205(48.4) \\
194(45.7) \\
287(67.7) \\
116(27.4) \\
21(4.9) \\
333(7.8) \\
143(33.7)\end{array}$ & $\begin{array}{c}64(30.8) \\
85(40.9) \\
9(4.3) \\
87(41.8) \\
112(53.9) \\
144(69.2) \\
53(25.5) \\
11(5.3) \\
16(7.7) \\
77(37.0)\end{array}$ & $\begin{aligned} & 55(25.5) \\
& 105(48.6) \\
& 16(7.4) \\
& 118(54.6) \\
& 82(38.0) \\
& 143(66.2) \\
& 63(29.2) \\
& 10(4.6) \\
& 17(7.9) \\
& 66(30.6)\end{aligned}$ & $\begin{array}{l}0.68 \\
0.95 \\
0.16\end{array}$ \\
\hline \multicolumn{6}{|l|}{ Other treatment characteristics } \\
\hline $\begin{array}{l}\text { Total node count } \\
\text { NAC/primary chemotherapyc }, n \\
\text { Adjuvant chemotherapyc, } n \\
\text { Salvage chemotherapycin } n\end{array}$ & & $\begin{array}{l}12(7-19) \\
29(6.8) \\
87(20.5) \\
55(13.0)\end{array}$ & $\begin{array}{l}12(7-19) \\
15(7.2) \\
34(16.4) \\
27(13.0)\end{array}$ & $\begin{array}{l}13(8-19) \\
14(6.5) \\
53(24.5) \\
28(13.0)\end{array}$ & $\begin{aligned} & 0.22 \\
& 0.77 \\
& 0.037^{\mathrm{a}} \\
> & 0.99\end{aligned}$ \\
\hline $\begin{array}{l}\text { Abbreviations: CIS = carcinoma in situ; NAC } \\
\text { or number (percent). } \\
\text { a A significant difference is indicated betwe } \\
\text { b Sum of co-morbidity score (including mal } \text { ' }{ }^{\text {Generally, chemotherapy was cisplatin-bas }}\end{array}$ & $\begin{array}{l}\text { o-adjuvant chemoth } \\
\text { he groups NLR } \geqslant 3 . \\
\text { ncy) and age score. } \\
\text { Patients with renal it }\end{array}$ & $\begin{array}{l}\text { NLR = neutrophil-to-lyn } \\
<3 \text {. } \\
\text { ency received carbopla }\end{array}$ & io; $W B C=$ white $b$ & All data presented as r & terquartile \\
\hline
\end{tabular}


clinical parameters including blood work results. Mortality data were obtained through the Princess Margaret Cancer Centre Cancer Registry. Institutional research ethics board approval was obtained.

Primary study exposure. The date of initiation of treatment for each patient was defined as the date of RC or the date of initiation of NAC for patients who received NAC. All patients were seen for medical assessment before the initiation of treatment. Generally, NLR was calculated using neutrophil and lymphocyte counts from a routine $\mathrm{CBC}$ with differentials performed on the same day as these visits (median of 6 days (interquartile range (IQR): 2-10 days) before initiation of treatment). Review of the pre-treatment clinic notes did not reveal any symptoms or signs of infections that may have influenced the NLR.

Outcome measures. Patients were generally seen at 6-8 weeks after the RC, and otherwise every 3-6 months early on for periodic physical examination, imaging to rule out hydronephrosis or tumour recurrence, and urethroscopy if indicated. Follow-up subsequently became less intensive based on individual physician's practice patterns and clinical suspicion. The outcome measures were recurrence-free survival (RFS), cancer-specific survival (CSS) and overall survival (OS) measured in months from the date of initial treatment.

Statistical analysis. Statistical analyses were performed using SAS v9.3 (SAS Institute Inc, Cary, NC, USA). Clinical characteristics were compared between patients with NLR values above and below the optimal cutoff point (see below) using the Wilcoxon rank-sum test for continuous variables and Pearson's $\chi^{2}$ test for categorical variables.

In the literature, there is heterogeneity in the NLR cutoff points used (Guthrie et al, 2013). Therefore, in order to determine the optimal cutoff point for clinical use, time-dependent receiveroperating characteristic (ROC) curves were created for each outcome measure at 12, 24, 36, 48 and 60 months (Heagerty and Zheng, 2005; Lu and Liu, 2006). NLR values between 1.5 and 6 were considered in 0.5 increments. The optimal NLR value for each outcome at a given time point was identified by minimising the distance from the ROC curve to the top left corner of the ROC plot (and thus optimising both sensitivity and specificity; Perkins and Schisterman, 2006).

Kaplan-Meier analyses with log-rank tests were then used to compare survival outcomes between patients with NLR values above $v s$ below the optimal cutoff point. To determine how NLR can influence risk stratification in the pre-treatment and postcystectomy settings among patients with localised disease (without evidence of nodal disease), we performed additional Kaplan-Meier analyses stratifying by clinical and pathological stage, respectively. In these analyses, patients receiving NAC or AC were excluded to have a more clear impression of how NLR values have an impact on the natural history of disease.

Univariate and multivariable Cox proportional hazards models were built for each survival outcome. Multivariable models adjusted for a priori defined patient-related risk factors (age, gender and Charlson comorbidity index), tumour-related variables (pathological T-stage and lymphovascular invasion), treatmentrelated parameters (year of $\mathrm{RC}$, use of NAC or AC and surgical margin status) and haematologic parameters (haemoglobin and platelet counts). AC was operationalised as a time-varying covariate to address survivor treatment bias (Austin et al, 2006). A robust sandwich covariance matrix estimator was used to account for clustering of outcomes by surgeon (Lin and Wei, 1989). The additional value to the models provided by NLR was evaluated using the likelihood-ratio test to compare models for each survival outcome with and without NLR. Statistical model assumptions, including the proportional hazards assumption, were tested (Hess, 1995).
In order to ensure that our use of a cutoff point did not introduce bias (Royston et al, 2006), we performed a sensitivity analysis analysing NLR as a continuous variable with logtransformation (because of its skewed distribution). Given that other studies have not included patients receiving NAC (Gondo et al, 2012), we also performed a sensitivity analysis excluding such patients. All tests were two-sided with $P$-values $<0.05$ considered statistically significant.
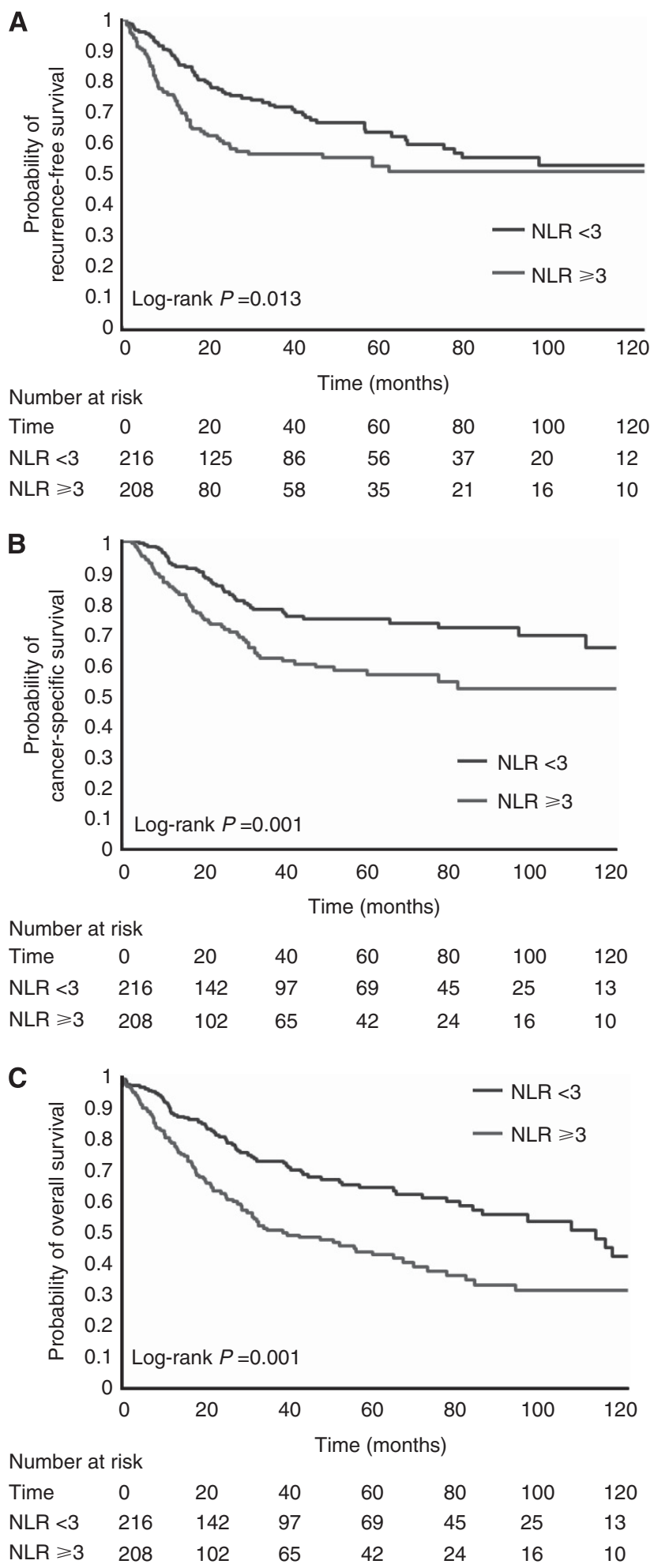

Figure 1. (A-C) Kaplan-Meier curves for recurrence-free survival (A), cancer-specific survival (B) and overall survival (C) for patients with an NLR $<3$ and $\geqslant 3$. 


\section{RESULTS}

The final study cohort consisted of 424 patients with a median follow-up of 58.4 months (IQR: 21.3-94.5 months). The cohort characteristics are described in Table 1. Overall, 138 patients (32.6\%) had cancer recurrence and 178 (42\%) died, of which 110 (25.9\%) died of UCB.

In time-dependent ROC curve analyses, an NLR cutoff point of 3 minimised the distance from the ROC curve to the top left of the plot for 14 out of 15 time points across the three outcome measures (Supplementary Table 1). Given that this cutoff point was among those used by other studies (Guthrie et al, 2013), we proceeded to use this as the optimal cutoff point in our study.
There were 216 (50.9\%) patients who had an NLR value $\geqslant 3$ (Table 1). These patients had significantly lower haemoglobin values, higher platelet counts and a higher Charlson comorbidity index and were less likely to receive AC. They were more likely to have pT3-4 disease $(53.9 \%$ vs 38\%); however, there was no significant difference in $\mathrm{pN}$ stage.

In univariate Kaplan-Meier analyses, NLR $\geqslant 3$ vs $<3$ was associated with increased probability of recurrence (5y-RFS: $53 \%$ vs $64 \%, \log$-rank $P=0.013$, Figure $1 \mathrm{~A}$ ), cancer-specific mortality (5yCSS: $57 \%$ vs 75\%, log-rank $P<0.001$, Figure 1B) and overall mortality (5y-OS: $43 \%$ vs 64\%, log-rank $P<0.001$; Figure $1 C$ ).

In univariate Cox models, an NLR $\geqslant 3$ was associated with increased risk of recurrence (hazard ratio $(\mathrm{HR})=1.53$, $95 \%$ confidence interval $(\mathrm{CI})=1.23-1.89, P<0.001$, Table 2),

Table 2. Cox proportional hazard models for recurrence-free survival

Unadjusted

Adjusted

\begin{tabular}{|c|c|c|c|c|}
\hline Parameter & $\mathrm{HR}(95 \% \mathrm{Cl})$ & $P$-value & $\operatorname{HR}(95 \% \mathrm{Cl})$ & $P$-value \\
\hline $\operatorname{NLR}(\geqslant 3$ vs $<3)$ & $1.53(1.23-1.89)$ & $<0.001$ & $1.49(1.12-2.00)$ & 0.007 \\
\hline Age & $1.01(1.00-1.02)$ & 0.22 & $1.00(0.98-1.03)$ & 0.93 \\
\hline Gender (female vs male) & $0.96(0.68-1.34)$ & 0.79 & $0.87(0.60-1.27)$ & 0.48 \\
\hline Charlson comorbidity index & $1.08(0.99-1.18)$ & 0.078 & $1.04(0.98-1.11)$ & 0.17 \\
\hline Haemoglobin (per 10 units) & $0.89(0.86-0.93)$ & $<0.001$ & $0.97(0.95-1.00)$ & 0.014 \\
\hline Platelets (per 100 units) & $1.24(1.10-1.38)$ & $<0.001$ & $1.17(1.10-1.25)$ & $<0.001$ \\
\hline T-stage (pT3-4 vs pT0-2) & $2.75(1.99-3.80)$ & $<0.001$ & $1.51(0.97-2.34)$ & 0.067 \\
\hline $\mathrm{N}$-Stage $(\mathrm{N}+$ vs No/Nx) & $3.01(2.68-3.38)$ & $<0.001$ & $2.16(1.82-2.57)$ & $<0.001$ \\
\hline Positive surgical margin & $2.07(1.33-3.22)$ & 0.001 & $1.99(1.28-3.10)$ & 0.002 \\
\hline Year of radical cystectomy & $1.00(0.97-1.03)$ & 0.93 & $1.00(0.97-1.03)$ & $>0.99$ \\
\hline Lymphovascular invasion & $2.91(1.88-4.52)$ & $<0.001$ & $1.82(1.13-2.95)$ & 0.014 \\
\hline NAC/primary chemotherapy & $0.93(0.43-2.01)$ & 0.86 & $0.90(0.41-1.97)$ & 0.79 \\
\hline Adjuvant chemotherapy & $1.56(1.21-2.00)$ & $<0.001$ & $0.89(0.55-1.42)$ & 0.61 \\
\hline
\end{tabular}

Abbreviations: $\mathrm{Cl}=$ confidence interval; $\mathrm{HR}=$ hazard ratio; $\mathrm{NAC}=$ neo-adjuvant chemotherapy; $\mathrm{NLR}=$ neutrophil-to-lymphocyte ratio.

Table 3. Cox proportional hazard models for cancer-specific survival

Unadjusted

Adjusted

\begin{tabular}{|c|c|c|c|c|}
\hline Parameter & $\mathrm{HR}(95 \% \mathrm{Cl})$ & $P$-value & $\mathrm{HR}(95 \% \mathrm{Cl})$ & $P$-value \\
\hline NLR ( $\geqslant 3$ vs $<3$ ) & $1.88(1.52-2.33)$ & $<0.001$ & $1.88(1.39-2.54)$ & $<0.001$ \\
\hline Age & $1.01(0.99-1.04)$ & 0.19 & $1.00(0.97-1.04)$ & 0.88 \\
\hline Gender (female vs male) & $1.17(0.95-1.46)$ & 0.14 & $1.10(0.87-1.40)$ & 0.41 \\
\hline Charlson Comorbidity Index & $1.16(1.06-1.28)$ & 0.002 & $1.16(1.09-1.24)$ & $<0.001$ \\
\hline Haemoglobin (per 10 units) & $0.87(0.82-0.91)$ & $<0.001$ & $0.96(0.92-0.99)$ & 0.013 \\
\hline Platelets (per 100 units) & $1.28(1.14-1.44)$ & $<0.001$ & $1.19(1.03-1.37)$ & 0.019 \\
\hline T-stage (pT3-4 vs pT0-2) & $3.12(2.18-4.47)$ & $<0.001$ & $1.65(1.06-2.56)$ & 0.026 \\
\hline $\mathrm{N}$-stage $(\mathrm{N}+$ vs $\mathrm{NO} / \mathrm{Nx})$ & $3.21(2.14-4.81)$ & $<0.001$ & $2.26(1.38-3.70)$ & 0.001 \\
\hline Positive surgical margin & $1.98(1.03-3.82)$ & 0.041 & $1.61(0.79-3.31)$ & 0.19 \\
\hline Year of radical cystectomy & $0.98(0.92-1.04)$ & 0.50 & $0.96(0.90-1.03)$ & 0.30 \\
\hline Lymphovascular invasion & $3.09(1.98-4.82)$ & $<0.001$ & $1.91(1.10-3.34)$ & 0.023 \\
\hline NAC/primary chemotherapy & $1.47(0.66-3.25)$ & 0.34 & $1.33(0.59-2.98)$ & 0.50 \\
\hline Adjuvant chemotherapy & $1.36(1.01-1.83)$ & 0.041 & $0.95(0.49-1.86)$ & 0.89 \\
\hline
\end{tabular}


Table 4. Cox proportional hazard models for overall survival

\begin{tabular}{|c|c|c|c|c|}
\hline \multirow[b]{2}{*}{ Parameter } & \multicolumn{2}{|c|}{ Unadjusted } & \multicolumn{2}{|c|}{ Adjusted } \\
\hline & $\mathrm{HR}(95 \% \mathrm{Cl})$ & $P$-value & $\mathrm{HR}(95 \% \mathrm{Cl})$ & $P$-value \\
\hline NLR $(\geqslant 3$ vs $<3)$ & $1.80(1.48-2.20)$ & $<0.001$ & $1.67^{\mathrm{a}}(1.17-2.39)$ & 0.005 \\
\hline Age & $1.03(1.02-1.05)$ & $<0.001$ & $1.01(0.99-1.03)$ & 0.20 \\
\hline Gender (female vs male) & $0.95(0.81-1.11)$ & 0.48 & $0.89(0.73-1.07)$ & 0.21 \\
\hline Charlson Comorbidity Index & $1.28(1.18-1.39)$ & $<0.001$ & $1.23(1.16-1.31)$ & $<0.001$ \\
\hline Haemoglobin (per 10 units) & $0.85(0.82-0.87)$ & $<0.001$ & $0.93(0.89-0.97)$ & $<0.001$ \\
\hline Platelets (per 100 units) & $1.21(1.11-1.31)$ & $<0.001$ & $1.21(1.14-1.28)$ & $<0.001$ \\
\hline T-stage (pT3-4 vs pT0-2) & $2.44(1.58-3.77)$ & $<0.001$ & $1.41(0.82-2.42)$ & 0.21 \\
\hline $\mathrm{N}$-stage $(\mathrm{N}+$ vs $\mathrm{N} 0 / \mathrm{Nx})$ & $2.22(1.67-2.94)$ & $<0.001$ & $1.59(1.14-2.22)$ & 0.006 \\
\hline Positive surgical margin & $2.00(0.99-4.04)$ & 0.054 & $1.83(0.94-3.60)$ & 0.078 \\
\hline Year of radical cystectomy & $0.97(0.93-1.01)$ & 0.15 & $0.95(0.92-0.98)$ & 0.004 \\
\hline Lymphovascular invasion & $2.65(2.06-3.41)$ & $<0.001$ & $2.08(1.18-3.68)$ & 0.012 \\
\hline NAC/primary chemotherapy & $1.44(0.67-3.06)$ & 0.35 & $1.31(0.81-2.13)$ & 0.28 \\
\hline Adjuvant chemotherapy & $0.91(0.62-1.33)$ & 0.61 & $0.84(0.48-1.49)$ & 0.55 \\
\hline
\end{tabular}

cancer-specific mortality $\quad(\mathrm{HR}=1.88, \quad 95 \% \quad \mathrm{CI}=1.52-2.33$, $P<0.001$, Table 3$)$ and overall mortality $(\mathrm{HR}=1.80,95 \%$ $\mathrm{CI}=1.48-2.20, P<0.001$, Table 4$)$.

Upon adjusting for confounders using multivariable models, NLR remained significantly associated with increased risk of recurrence $(\mathrm{HR}=1.49,95 \% \mathrm{CI}=1.12-2, P=0.007$, Table 2), cancer-specific mortality $(\mathrm{HR}=1.88, \quad 95 \% \quad \mathrm{CI}=1.39-2.54$, $P<0.001$, Table 3 ) and overall mortality (average $\mathrm{HR}=1.67$, $95 \% \mathrm{CI}=1.17-2.39, P=0.005$, Table 4$)$. Of note, the proportional hazards assumption was satisfied for the models for RFS and CSS but not for OS. This would suggest that the HR for the association between NLR and OS is not constant but varies as a function of time. Therefore, the HR presented in Table 4 represents an average value across the study period. As others have presented such findings (Lipscombe et al, 2013), Figure 2 shows how the HR changes as a function of time from initial treatment (with the corresponding model that includes an NLR $\times$ time interaction term shown in Supplementary Table 2). Neutrophil-to-lymphocyte ratio has the strongest association with increased risk of overall mortality early on and then gradually decreases. This association remained statistically significant up to $\sim 50$ months from the start of treatment.

Associations between NLR and increased risk of adverse survival outcomes remained statistically significant when NLR was analysed as a log-transformed continuous variable, as well as in the sensitivity analysis excluding patients who received NAC (data not shown). Using the likelihood-ratio test and comparing multivariable Cox models with and without NLR, it was determined that NLR significantly improved models for RFS $(P=0.013)$, CSS $(P=0.001)$ and $\mathrm{OS}(P=0.003)$.

Lastly, we performed exploratory analyses to assess the potential prognostic impact of using NLR when risk stratifying patients into two scenarios. For these analyses, patients receiving NAC or AC were excluded to better reflect the natural history of disease and avoid confounding from adjunctive treatment. The first scenario assessed patients without clinical evidence of nodal disease (cN0), where risk stratification may guide initial management in the pretreatment setting. We sought to determine whether NLR further stratified patients beyond their clinical stage, analysing its impact

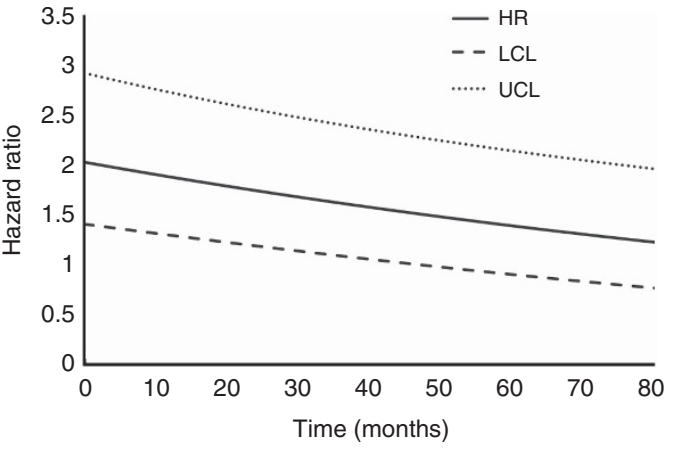

Figure 2. Instantaneous hazard ratio vs time with corresponding confidence intervals for OS LCL = lower $95 \%$ confidence limit, $\mathrm{UCL}=$ upper $95 \%$ confidence limit.

on clinically NMI UCB vs MI UCB disease subgroups. In these Kaplan-Meier analyses (Figures 3A-C), NLR added valuable prognostic information. Patients with clinical NMI UCB appeared to separate into two groups, with those with clinical NMI UCB and NLR $\geqslant 3$ manifesting survival outcomes comparable to clinical MI UCB. The second scenario was the postoperative setting among patients who were pN0 (where a decision must be made regarding the use of AC). NLR further stratified patients within pT-stage categories (Figures $4 \mathrm{~A}-\mathrm{C}$ ). Notably among patients with organconfined (pT0-pT2) disease, NLR identified a subset of patients who were at increased risk of adverse oncologic outcomes.

\section{DISCUSSION}

The host inflammatory response has gained increasing attention in oncology research. Infiltrating cells of the immune system are constituents of virtually all neoplasms (Hanahan and Weinberg, 2011). While initially thought to represent an antitumoural response, immune cells, particularly those of the innate immune system, also exhibit effects that promote carcinogenesis and 
A

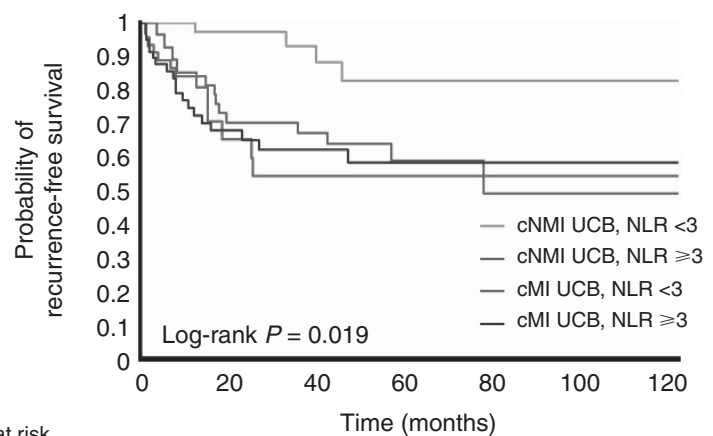

Number at risk

Time

CNMI UCB, NLR $<3 \quad 4$

CNMI UCB, NLR $\geqslant 3$

CMI UCB, NLR $<3$

cMI UCB, NLR $\geqslant 3$

B

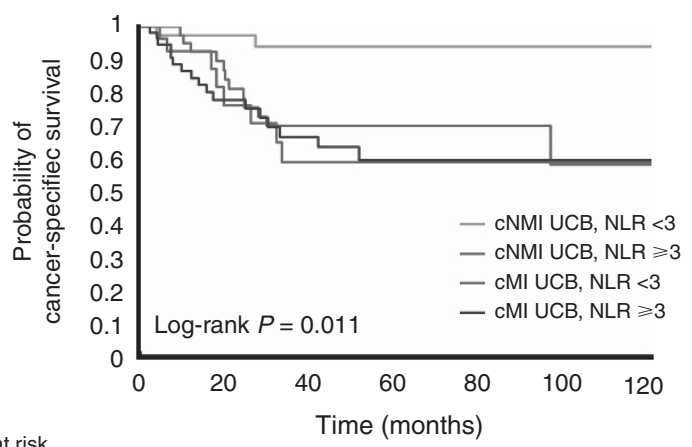

\begin{tabular}{|c|c|c|c|c|c|c|c|}
\hline \multicolumn{8}{|l|}{ Number at risk } \\
\hline Time & 0 & 20 & 40 & 60 & 80 & 100 & 120 \\
\hline cNMI UCB, NLR <3 & 44 & 32 & 21 & 14 & 10 & 5 & 2 \\
\hline cNMI UCB, NLR $\geqslant 3$ & 28 & 14 & 9 & 4 & 2 & 2 & 2 \\
\hline cMI UCB, NLR <3 & 51 & 31 & 23 & 16 & 8 & 5 & 2 \\
\hline $\mathrm{cMI} \cup \mathrm{CB}, \mathrm{NLR} \geqslant 3$ & 65 & 31 & 22 & 14 & 7 & 5 & 2 \\
\hline
\end{tabular}

c

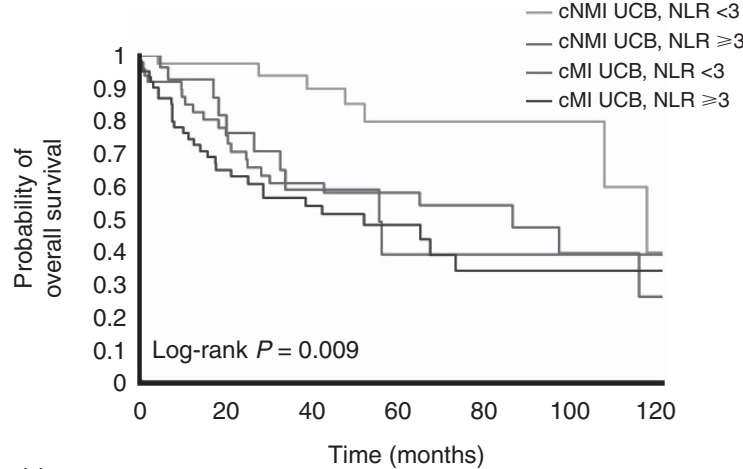

Number at risk

$\begin{array}{llllllll}\text { Time } & 0 & 20 & 40 & 60 & 80 & 100 & 120\end{array}$

$\begin{array}{llllllll}\mathrm{CNMI} \text { UCB, NLR }<3 & 44 & 30 & 18 & 12 & 9 & 3 & 1\end{array}$

$\begin{array}{llllllll}\mathrm{CNMI} U \mathrm{UCB}, \mathrm{NLR} \geqslant 3 & 28 & 12 & 8 & 3 & 2 & 2 & 2\end{array}$

$\begin{array}{llllllll}\mathrm{CMI} U \mathrm{CB}, \mathrm{NLR}<3 & 51 & 25 & 20 & 10 & 5 & 4 & 2\end{array}$

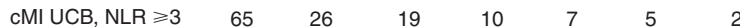

Figure 3. (A-C) Kaplan-Meier curves for recurrence-free survival (A), cancer-specific survival (B) and overall survival (C) for patients without clinical evidence of nodal disease ( $\mathrm{cNO}$ ) and clinically NMI bladder cancer $(\mathrm{BC})$ or $\mathrm{MI} B C$ and an NLR $<3$ or $\geqslant 3$, respectively.

cancer progression (Grivennikov et al, 2010; Hanahan and Weinberg, 2011). Proposed mechanisms include increased supply of growth factors, survival factors, pro-angiogenic factors, extracellular matrix-modifying enzymes (which can facilitate invasion and metastasis) and inductive signals that may lead to epithelial-to-mesenchymal transition (Hanahan and Weinberg, 2011). Thus, there is a biological rationale for using NLR, the ratio of circulating neutrophils (immune cells of the innate system) to lymphocytes (immune cells of the adaptive system), as a measure
A

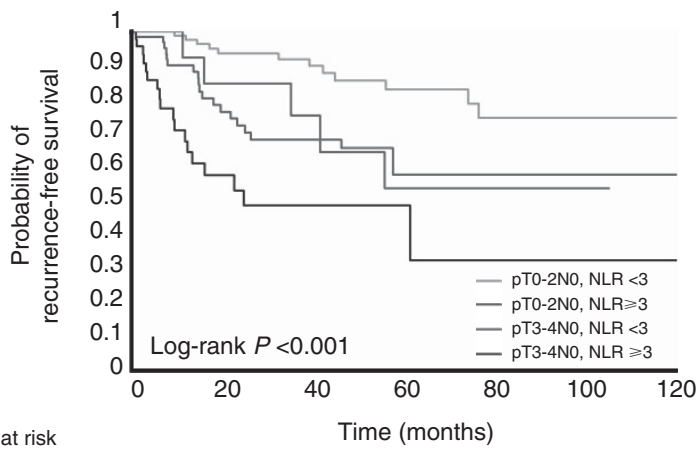

\begin{tabular}{lccccccc} 
Number at risk & \multicolumn{7}{c}{ Time (months) } \\
Time & 0 & 20 & 40 & 60 & 80 & 100 & 120 \\
pT0-2N0, NLR $<3$ & 100 & 67 & 45 & 29 & 18 & 8 & 4 \\
pT0-2NO, NLR $\geqslant 3$ & 66 & 38 & 28 & 15 & 10 & 7 & 5 \\
pT3-4NO, NLR $<3$ & 18 & 11 & 8 & 4 & 4 & 2 & 0 \\
pT3-4NO, NLR $\geqslant 3$ & 49 & 14 & 8 & 3 & 1 & 1 & 1
\end{tabular}

B

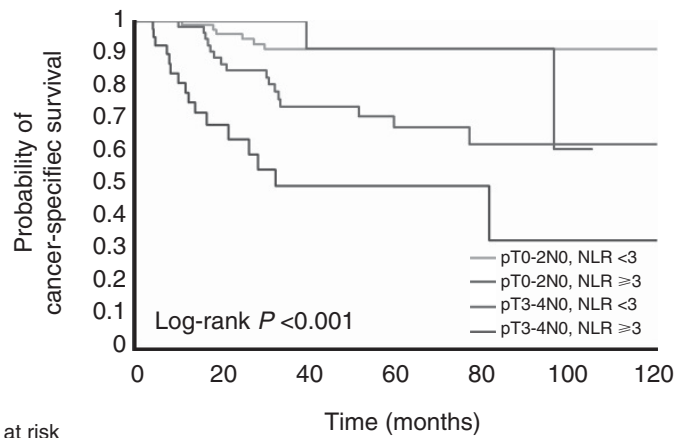

Number at risk

$\begin{array}{lrrrcccc}\text { Time } & 0 & 20 & 40 & 60 & 80 & 100 & 120 \\ \text { pTO-2N0, NLR }<3 & 100 & 70 & 49 & 37 & 22 & 11 & 5 \\ \text { pT0-2N0, NLR } \geqslant 3 & 66 & 45 & 30 & 20 & 12 & 7 & 5 \\ \text { pT3-4N0, NLR }<3 & 18 & 14 & 11 & 6 & 6 & 2 & 0 \\ \text { pT3-4N0, NLR } \geqslant 3 & 49 & 16 & 10 & 6 & 3 & 2 & 2\end{array}$

pT $3-4$ NO, NLR $\geqslant 3 \quad 49$

C

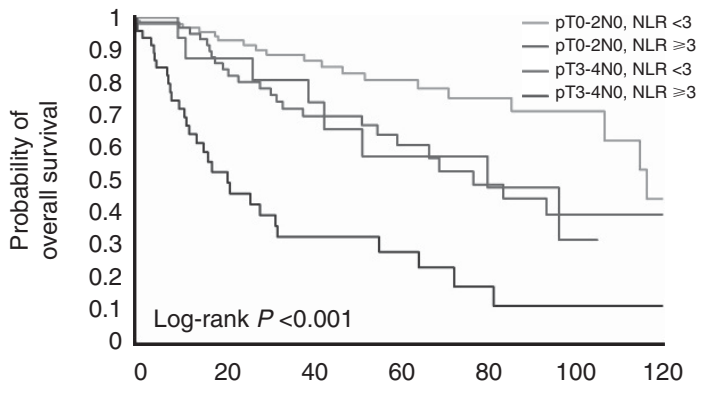

Number at risk

Time

pTO-2NO, NLR $<3$

pTO-2NO, NLR $\geqslant 3$

pT3-4NO, NLR $<3$

pT $3-4 N 0, N L R \geqslant 3$

$\begin{array}{ccccccc}0 & 20 & 40 & 60 & 80 & 100 & 120 \\ 100 & 70 & 49 & 37 & 22 & 11 & 5 \\ 66 & 45 & 30 & 20 & 12 & 7 & 5 \\ 18 & 14 & 11 & 6 & 6 & 2 & 0 \\ 49 & 16 & 10 & 6 & 3 & 2 & 2\end{array}$

Figure 4. (A-C) Kaplan-Meier curves for recurrence-free survival (A), cancer-specific survival (B) and overall survival (C) for patients with organ-confined bladder cancer (pT0-2 pN0) or non-organ-confined bladder cancer ( $\mathrm{pT} 3-4 \mathrm{pNO}$ ) and an NLR $<3$ or $\geqslant 3$, respectively.

of the systemic host response when evaluating the association between inflammation and cancer outcomes.

The prognostic role of NLR has been evaluated in numerous epidemiologic studies of various cancer types. Higher NLR has been found to be consistently associated with more advanced stage and more aggressive tumour behaviour (Guthrie et al, 2013; Templeton et al, 2014). However, data regarding the association of NLR and prognosis for UCB after RC are still scarce. To date, only three small studies have been published in this population (Gondo et al, 2012; Demirtas et al, 2013; Krane et al, 2013). Gondo et al (2012) were the first to describe an association between higher 
NLR (>2.5) and CSS in a cohort of 189 patients undergoing RC. Demirtas et al (2013) (201 patients) reported no association between NLR $(>2.5)$ and OS, whereas Krane et al (2013) (68 patients) found that an elevated NLR $(>2.5)$ was an independent predictor of extravesical disease and worse OS. In the latter study, however, 10 patients received NAC and it is unclear how their calculated NLR based on immediate preoperative blood work may have been affected.

To the best of our knowledge, our study has the largest sample size investigating the independent prognostic ability of NLR in patients undergoing RC for UCB. It is the first of its kind to show that pre-treatment NLR is an independent prognostic factor for RFS, CSS and OS. Among patients receiving NAC, we used CBCs collected before the initiation of chemotherapy to eliminate this potential confounder. In addition, a sensitivity analysis excluding patients receiving NAC was performed to confirm robustness of the findings.

There is heterogeneity in reported thresholds used to define an elevated NLR in the literature (range 2-7.7; Templeton et al, 2014). This may reflect variations in the host response for different disease sites and stages, or may reflect the different approaches used when determining cutoff values. Not all studies used an accepted method for cutoff point determination, and in some instances the rationale for the cutoff point decision was not described (Templeton et al, 2014). All three previously mentioned studies in the RC population used an optimal NLR cutoff point of 2.5 (Gondo et al, 2012; Demirtas et al, 2013; Krane et al, 2013). Gondo et al (2012) used the cutoff point that generated the lowest $P$-value in Kaplan-Meier analyses. It is unclear, however, whether this cutoff point was associated with optimal sensitivity and specificity for adverse oncologic outcomes in their study population. One of the other studies chose 2.5 as their cutoff point for consistency with Gondo et al (2012) (Krane et al, 2013), whereas the third study did not elaborate on the rationale for their cutoff point value (Demirtas et al, 2013).

We used time-dependent ROC curves to determine the optimal cutoff point for NLR. Whereas ROC curves are conventionally used for binary outcomes to identify points of optimal sensitivity and specificity, this approach was adapted for survival analyses (Heagerty and Zheng, 2005; Lu and Liu, 2006). In our study, 3 was determined optimal cutoff point. We felt that it was important to identify an a priori optimal cutoff point both for practical purposes, and to minimise bias. Even so, there is likely a continuous association between NLR and risk of adverse oncologic outcomes. This warranted a sensitivity analysis using NLR as a logtransformed continuous variable to ensure that we did not introduce any cutoff point bias (Royston et al, 2006).

Lastly, our exploratory analyses indicate that NLR may better risk-stratify patients in the pre- and postoperative settings in order to guide treatment strategies. In patients with clinically NMI UCB, there is a high risk of under-staging and a high risk of disease progression to MI UCB (Shariat et al, 2007; Thomas et al, 2012; Chamie et al, 2013). The NLR may be helpful to identify patients most likely to benefit from early RC. Similarly, NLR may improve postoperative risk stratification to guide the use of AC. However, this was not the primary objective of this study and further work is needed to identify the clinical scenarios in which NLR may be helpful.

There are limitations to our study. First, this is a retrospective, single-institution observational study. Second, our study included patients across a long recruitment period, during which practice patterns might have changed. We addressed this by including year of cystectomy in the multivariable model. Third, we did not measure NLR after RC and therefore cannot investigate whether post-RC improvement of NLR has a predictive value. Finally, we are unable to determine whether the outcomes following NAC or $\mathrm{AC}$ are different among those patients with high $v s$ low NLR because of the limited number of patients receiving NAC or AC in our cohort.

In conclusion, NLR is an inexpensive haematologic test based on commonly measured parameters that predicts RFS, CSS and OS in patients with UCB undergoing RC, independent of wellestablished patient-related and tumour-related predictors. Whereas our results suggest that NLR may have a role as a prognostic biomarker in the pre-RC and post-RC settings, further studies are needed to maximise the clinical utility of NLR.

\section{REFERENCES}

Austin PC, Mamdani MM, van Walraven C, Tu JV (2006) Quantifying the impact of survivor treatment bias in observational studies. J Eval Clin Prac 12(6): 601-612.

Canter D, Long C, Kutikov A, Plimack E, Saad I, Oblaczynski M, Zhu F, Viterbo R, Chen DY, Uzzo RG, Greenberg RE, Boorjian SA (2011) Clinicopathological outcomes after radical cystectomy for clinical T2 urothelial carcinoma: further evidence to support the use of neoadjuvant chemotherapy. BJU Int 107(1): 58-62.

Chamie K, Litwin MS, Bassett JC, Daskivich TJ, Lai J, Hanley JM, Konety BR, Saigal CS. Urologic Diseases in America P (2013) Recurrence of high-risk bladder cancer: a population-based analysis. Cancer 119(17): 3219-3227.

Clark PE, Agarwal N, Biagioli MC, Eisenberger MA, Greenberg RE, Herr HW, Inman BA, Kuban DA, Kuzel TM, Lele SM, Michalski J, Pagliaro LC, Pal SK, Patterson A, Plimack ER, Pohar KS, Porter MP, Richie JP, Sexton WJ, Shipley WU, Small EJ, Spiess PE, Trump DL, Wile G, Wilson TG, Dwyer M, Ho M. National Comprehensive Cancer N (2013) Bladder cancer. J Natl Compr Canc Netw 11(4): 446-475.

Demirtas A, Sabur V, Akinsal EC, Demirci D, Ekmekcioglu O, Gulmez I, Tatlisen A (2013) Can neutrophil-lymphocyte ratio and lymph node density be used as prognostic factors in patients undergoing radical cystectomy? TheScientificWorldJournal 2013: 703579.

Ficarra V, Dalpiaz O, Alrabi N, Novara G, Galfano A, Artibani W (2005) Correlation between clinical and pathological staging in a series of radical cystectomies for bladder carcinoma. BJU Int 95(6): 786-790.

Gakis G, Efstathiou J, Lerner SP, Cookson MS, Keegan KA, Guru KA, Shipley WU, Heidenreich A, Schoenberg MP, Sagaloswky AI, Soloway MS, Stenzl A. International Consultation on Urologic Disease-European Association of Urology Consultation on Bladder C (2013) ICUD-EAU International Consultation on Bladder Cancer 2012: radical cystectomy and bladder preservation for muscle-invasive urothelial carcinoma of the bladder. Eur Urol 63(1): 45-57.

Gondo T, Nakashima J, Ohno Y, Choichiro O, Horiguchi Y, Namiki K, Yoshioka K, Ohori M, Hatano T, Tachibana M (2012) Prognostic value of neutrophil-to-lymphocyte ratio and establishment of novel preoperative risk stratification model in bladder cancer patients treated with radical cystectomy. Urology 79(5): 1085-1091.

Grivennikov SI, Greten FR, Karin M (2010) Immunity, inflammation, and cancer. Cell 140(6): 883-899.

Guthrie GJ, Charles KA, Roxburgh CS, Horgan PG, McMillan DC, Clarke SJ (2013) The systemic inflammation-based neutrophil-lymphocyte ratio: experience in patients with cancer. Cri Rev Oncol Hematol 88(1): 218-230.

Hanahan D, Weinberg RA (2011) Hallmarks of cancer: the next generation. Cell 144(5): 646-674.

Heagerty PJ, Zheng Y (2005) Survival model predictive accuracy and ROC curves. Biometrics 61(1): 92-105.

Hess KR (1995) Graphical methods for assessing violations of the proportional hazards assumption in Cox regression. Stat Med 14(15): 1707-1723.

Krane LS, Richards KA, Kader AK, Davis R, Balaji KC, Hemal AK (2013) Preoperative neutrophil/lymphocyte ratio predicts overall survival and extravesical disease in patients undergoing radical cystectomy. J Endourol 27(8): 1046-1050.

Leow JJ, Martin-Doyle W, Rajagopal PS, Patel CG, Anderson EM, Rothman AT, Cote RJ, Urun Y, Chang SL, Choueiri TK, Bellmunt J (2013) Adjuvant chemotherapy for invasive bladder cancer: a 2013 updated systematic review and meta-analysis of randomized trials. Eur Urol; e-pub ahead of print 28 August 2013; doi:10.1016/j.eururo.2013.08.033.

Lin D, Wei LJ (1989) The robust inference for the Cox proportional hazards model. J Am Stat Assoc 84(408): 1074-1078. 
Lipscombe LL, Chan WW, Yun L, Austin PC, Anderson GM, Rochon PA (2013) Incidence of diabetes among postmenopausal breast cancer survivors. Diabetologia 56(3): 476-483.

Lu L, Liu C (2006) Using the Time Dependent ROC Curve to Build Better Survival Model in SAS. NorthEast SAS Users Group Conferences (NESUG); Philadelphia, 17-20 September 2006.

Meeks JJ, Bellmunt J, Bochner BH, Clarke NW, Daneshmand S, Galsky MD, Hahn NM, Lerner SP, Mason M, Powles T, Sternberg CN, Sonpavde G (2012) A systematic review of neoadjuvant and adjuvant chemotherapy for muscle-invasive bladder cancer. Eur Urol 62(3): 523-533.

Perkins NJ, Schisterman EF (2006) The inconsistency of "optimal" cutpoints obtained using two criteria based on the receiver operating characteristic curve. Am J Epidemiol 163(7): 670-675.

Proctor MJ, McMillan DC, Morrison DS, Fletcher CD, Horgan PG, Clarke SJ (2012) A derived neutrophil to lymphocyte ratio predicts survival in patients with cancer. Br J Cancer 107(4): 695-699.

Raj GV, Karavadia S, Schlomer B, Arriaga Y, Lotan Y, Sagalowsky A, Frenkel E (2011) Contemporary use of perioperative cisplatin-based chemotherapy in patients with muscle-invasive bladder cancer. Cancer 117(2): 276-282.

Royston P, Altman DG, Sauerbrei W (2006) Dichotomizing continuous predictors in multiple regression: a bad idea. Stat Med 25(1): 127-141.
Shariat SF, Palapattu GS, Karakiewicz PI, Rogers CG, Vazina A, Bastian PJ, Schoenberg MP, Lerner SP, Sagalowsky AI, Lotan Y (2007) Discrepancy between clinical and pathologic stage: impact on prognosis after radical cystectomy. Eur Urol 51(1): 137-149.

Sternberg CN, Bellmunt J, Sonpavde G, Siefker-Radtke AO, Stadler WM, Bajorin DF, Dreicer R, George DJ, Milowsky MI, Theodorescu D, Vaughn DJ, Galsky MD, Soloway MS, Quinn DI. International Consultation on Urologic Disease-European Association of Urology Consultation on Bladder C (2013) ICUD-EAU International Consultation on Bladder Cancer 2012: chemotherapy for urothelial carcinomaneoadjuvant and adjuvant settings. Eur Urol 63(1): 58-66.

Templeton AJ, McNamara MG, Šeruga B, Vera-Badillo FE, Aneja P, Ocaña A, Leibowitz-Amit R, Sonpavde G, Knox JJ, Tran B, Tannock IF, Amir E (2014) Prognostic role of neutrophil-to-lymphocyte ratio in solid tumors: a systematic review and meta-analysis. J Natl Cancer Inst 106(6): dju124.

Thomas F, Rosario DJ, Rubin N, Goepel JR, Abbod MF, Catto JW (2012) The long-term outcome of treated high-risk nonmuscle-invasive bladder cancer: time to change treatment paradigm? Cancer 118(22): 5525-5534.

This work is published under the standard license to publish agreement. After 12 months the work will become freely available and the license terms will switch to a Creative Commons AttributionNonCommercial-Share Alike 3.0 Unported License.

Supplementary Information accompanies this paper on British Journal of Cancer website (http://www.nature.com/bjc) 